\section{Lipids and schizophrenia}

\author{
NICHOLAS P. WALKER, HELEN C. FOX and LAWRENCE J. WHALLEY
}

Lipid neurochemistry is now an important focus in schizophrenia research. Reports of abnormalities in brain lipids in schizophrenia appear widely in the medical and lay press. Disordered brain fatty acid metabolism was first postulated to play a part in the pathophysiology of schizophrenia by Horrobin (1977) and lately there is evidence that new treatments could follow (Mellor et al, 1996; Puri et al, 1998). At first glance the unlikeliness of this approach is striking. Lipids are ubiquitous; they are essential to life, forming the membranes that bound individual cells. Two polyunsaturated fatty acids (PUFAs) are essential components of the human diet. These essential fatty acids are linoleic acid and $\alpha$-linolenic acid. Each has 18 carbon atoms. They head up two series of PUFAs through repeated elongation and desaturation (Fig.
Linoleic acid

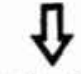

$\gamma$-Linolenic acid

凤

Dihomo- $\gamma$-linolenic acid

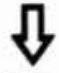

Arachidonic acid<smiles>C1=CC1</smiles>

Adrenic acid

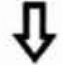

Tetracosatetraenoic acid<smiles>C1=CC=C1</smiles>

Tetracosapentaenoic acid

Docosapentaenoic acid
1). All PUFAs are stored in phospholipids, such as phospatidyl ethanolamine, within cell membranes. They play a role in membrane structure and can be released by phospholipase $\mathrm{A}_{2}$ and related enzymes. These free PUFAs are precursors of many compounds important to the healthy function of nervous tissue, including glycolipids, cholesterol esters, sphingolipids, thromboxanes, leucotrienes and prostaglandins (Fig. 2 ). The compound produced depends upon the combination of the specific PUFA and the enzyme pathway. Polyunsaturated fatty acids constitute about $20 \%$ of the dry weight of brain and, of these, arachidonic acid and docosahexaenoic acid account for about $70 \%$.

Some severe neurological syndromes are determined by genetic abnormalities in enzymes that degrade or remodel brain lipids. Major dysfunction of lipid metabolism reduces life expectancy. The childhood cerebral form of adrenoleucodystrophy is symptomatic early, and death supervenes two years from onset. Milder forms of adrenoleucodystrophy have a later onset, a more chronic course and are compatible with life but cerebral function is impaired. They are of relevance to schizophrenia research. Variants of the leucodystrophies respond to Lorenzo oil (Moser, 1993), a rape-seed derivative and one of the first synthetic "nutripharms' (nutrients used pharmacologically, usually in ultra-high dosage). By analogy, an apparently relatively minor lipid dysfunction could lead to the symptoms of schizophrenia. Nutripharms are 'naturally' appealing to the general public and to patients. A Mental Health Foundation survey (reported by D. Brindle, in The Guardian, 22 February 1997) revealed that $85-97 \%$ of psychiatric patients found alternative therapies (including dietary and natural supplements) helpful, compared with $70 \%$ for drug treatment and electroconvulsive therapy. The potential market for nutripharms in psychiatry may have hastened investigation of lipid dysfunction hypotheses in schizophrenia.

\section{ABNORMAL LIPID METABOLISM IN SCHIZOPHRENIA}

There is some evidence of abnormal lipid metabolism in schizophrenia beyond what is attributable to drugs, diet and lifestyle. Horrobin (1977) postulated that schizophrenia could arise from a deficiency within the eicosanoid system in which prostaglandins are major constituents. He reviewed much circumstantial support for his hypothesis. For example, he cited historical evidence that subjects with schizophrenia, compared with those not suffering from schizophrenia, are less likely to display a significant inflammatory reaction to typhoid vaccination. Prostaglandins and related compounds are potent substances that are difficult to study in vivo in schizophrenia because of their extremely short half-lives and their presence at very low concentrations. They are synthesised and degraded at their site of action. Direct measurement in the central nervous system is impractical, except at post-mortem. Horrobin et al (1991) analysed the phospholipid composition of frontal and cerebellar cortex at necropsy in a comparison

desaturation (desaturase, DS) and elongation (elongase, EL). 
Membrane Phospholipid

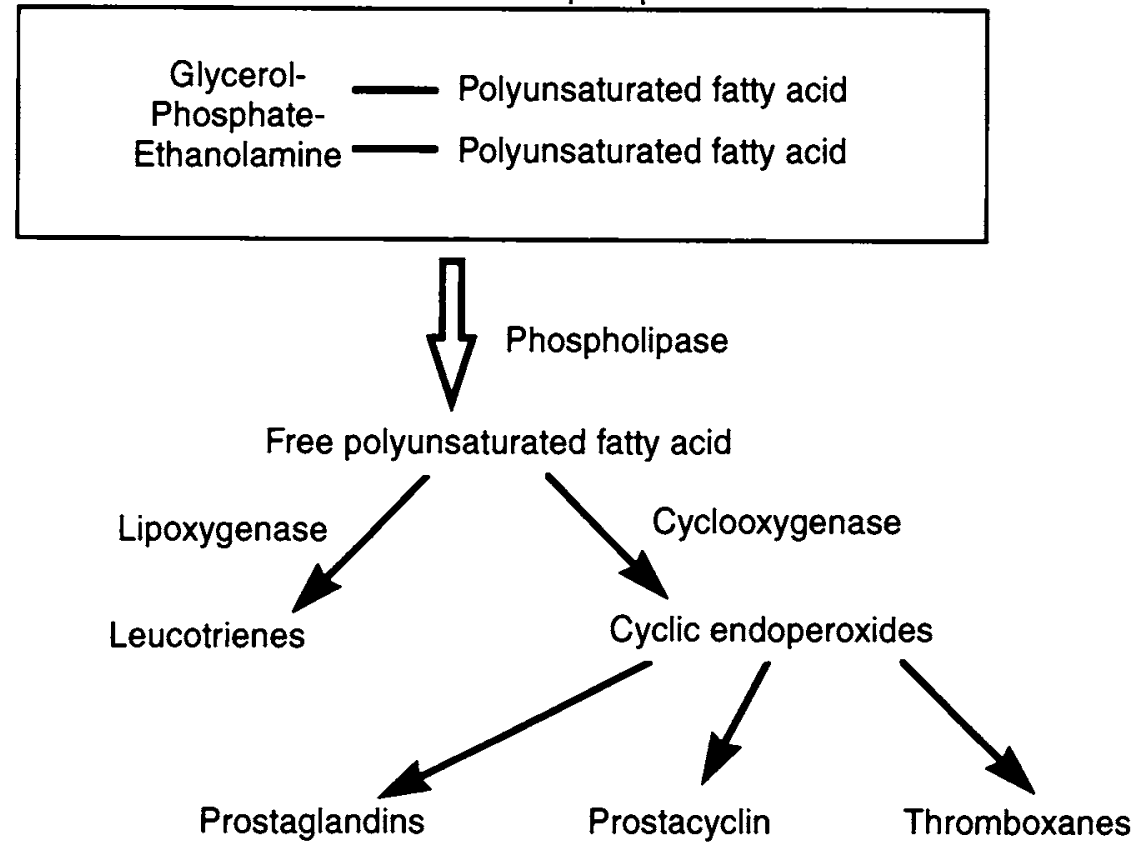

Fig. 2 Polyunsaturated fatty acid release from storage in membrane phospholipids and involvement in eicosanoid synthesis.

between seven subjects with schizophrenia and seven healthy controls. Arachidonic acid concentrations in the frontal lobes were significantly lower in the subjects with schizophrenia. Cerebellar tissue showed no significant differences. Horrobin et al (1991) argued that the patients' cerebellar results controlled for dietary and medication effects. Post-mortem artefacts could not be excluded and storage conditions were not uniform.

There are two approaches in vivo to the measurement of brain lipid metabolism: bioassay and measurement in peripheral cell models. Expediency and convenience have forced choices for modelling central nervous system structures in this way, but there is some evidence to support the use of a red blood cell membrane model to study lipid pathology in psychiatric disease. Makrides et al (1994) measured PUFA concentrations in cell membranes from cerebral cortex and erythrocytes from infants at necropsy to assess the effects of breast- and bottle-feeding. They found a significant correlation between the lipid profiles of cerebral cortex and erythrocyte. Caution is required. Carlson et al (1986) studied red cell and neuronal membrane PUFAs in newborn rats under a variety of dietary conditions. Although changes in membrane PUFA concentrations were not significantly different between the two cell types, absolute concentrations did differ significantly.

\section{LIPID METABOLISM MEASURED BY BIOASSAY}

Early studies of prostaglandin function in schizophrenia used bioassay. A prostaglandin-mediated system is measured indirectly by stimulating 'upstream' and recording the effect 'downstream'. Abdulla \& Hamadah (1975) treated platelet suspensions with adenosine bisphosphate, which stimulates arachidonic acid release from phospholipids. Platelet enzymes convert arachidonic acid to prostaglandins in normal subjects inducing platelet aggregation; in schizophrenia aggregation is reduced. This finding fits with the hypothesis that prostaglandin activity is reduced in schizophrenia.

\section{LIPID METABOLISM MEASURED IN THE PERIPHERY}

Measurement of prostaglandin precursors in peripheral cell models provides an indirect measurement of prostaglandins. The assumption underlying this method is that increases (or decreases) in precursor lead to increased (or decreased) synthesis. This has some validity in terms of biochemical equilibria. It explains the action of medicines such as L-dopa in Parkinson's disease. Fischer et al (1992) compared platelet membrane PUFA concentrations in 16 subjects with schizophrenia and six controls and found lower concentrations in those with schizophrenia. They found a strong negative correlation between PUFA concentrations and antipsychotic drug dose, and postulated that lowered lipids in subjects with schizophrenia may be due to antipsychotic medication. However, this part of their analysis lacked the power to establish a relationship.

Glen et al (1994) compared 68 subjects with schizophrenia and 259 controls and found that erythrocyte membrane arachidonic acid and docosahexaenoic acid were lower in those with schizophrenia. They also reported that their cohort with schizophrenia fell into two groups; there was a bimodal distribution of PUFAs, not present in the control group. Approximately threequarters of the subjects with schizophrenia shared the control group's Gaussian distribution, while the remaining quarter showed a distinct, lower distribution pattern. Peet et al (1994) reported similar findings but they went one step further; they looked at PUFA peroxidation products. These were elevated in the subjects with schizophrenia and were correlated negatively with arachidonic acid. They suggested that lowered arachidonic acid may be due to increased breakdown. None of these reports included data on diet or medication.

\section{LIPID METABOLISM MEASURED IN BRAIN}

The most exciting developments in lipid neurochemistry to date are direct measurements of phospholipid in the living brain using functional magnetic resonance imaging (fMRI), specifically ${ }^{31} \mathrm{P}-\mathrm{MRI}$. Pettegrew et al (1991) used fMRI to examine brain phospholid metabolism. They compared a first-episode drug-free cohort with schizophrenia with a control group and found significantly raised phosphodiester concentrations, suggesting increased breakdown by phospholipase $A_{2}$, and decreased phosphomonoesters, which could suggest lowered phospholipid synthesis in schizophrenia. Stanley et al (1995) reported similar findings with drug-treated and drug-free groups of subjects with schizophrenia and normal controls. These enquiries involved small numbers and studies were restricted 
to the dorsolateral prefrontal cortex. Although the results are not open to simple interpretation, they remain landmark studies of brain lipid metabolism in schizophrenia. They provide consistent results, apparently independent of drug effect, and have encouraged many research groups to begin studies on lipid metabolism in psychiatric disorders, especially on possible causes of excess phospholipid breakdown.

\section{RELEVANCE TO CAUSAL HYPOTHESES OF SCHIZOPHRENIA}

\section{Genetics and neurodevelopment}

There are likely to be large genetic and environmental contributions to the causes of schizophrenia and examination of the effects of specific genes on neurodevelopment may prove productive (Jones \& Murray, 1991). Gene-diet interaction early in neurodevelopment (McGill et al, 1996) may uncover genetically determined abnormalities in lipid metabolism of causal importance in the later onset of schizophrenia. Neurodevelopmental studies underline the importance of an adequate dietary content of essential fatty acids for the integrity of brain structures (Crawford, 1993). Maternal arachidonic acid and docosahexaenoic acid are important determinants of foetal brain size, possibly through their effects on synaptic outgrowth and remodelling (Hess et al, 1993). In the case of two PUFAs, eicosapentaenoic acid and docosahexaenoic acid, adequate dietary content contributes to the functional integrity of the brain and may explain the putative advantages of breast milk (which contains both eicosapentaenoic acid and docosahexaenoic acid) over formula feeds (which do not) in both verbal and performance intelligence (Lucas et al, 1992).

In prostaglandin synthesis, phospholipase $A_{2}$ is the rate-limiting enzyme. It catalyses the release of PUFAs from phospholipids and is involved in synaptic outgrowth. Inhibition of phospholipase $\mathrm{A}_{2}$ delays neuritic outgrowth, and administration of phospholipase $A_{2}$ will prevent neuritic retraction (Smalheiser et al, 1996). Mixed reports suggest either increased (Gattaz et al, 1987) or normal (Albers et al, 1993) phospholipase $A_{2}$ activity in subjects with schizophrenia compared to controls. Such variations require careful investigation. Ross et al (1997) addressed these inconsistencies and asked whether dif- ferent investigators had measured different subtypes of phospholipase $A_{2}$. They found that calcium-independent phospholipase $A_{2}$ activity was increased in the group with schizophrenia but calcium-dependent activity was normal, thus explaining the discrepancies of previous studies. These findings may also prove relevant to the role of phospholipase $A_{2}$ in synaptic remodelling; Negre-Aminou et al (1996) demonstrated that calcium-independent (but not calcium-dependent) phospholipase $A_{2}$ is enriched in the nerve growth cone compared to other parts of the foetal brain. Huttenlocher (1979) reported late maturational changes in normal brain, which occur mostly in late childhood and early adolescence and result in a marked reduction in synaptic density. These stages in neurodevelopment, when cellular changes occur at a high rate over a short time, represent 'critical periods'. During critical periods, the nervous system is likely to be especially susceptible to environmental and other insults. Increased phospholipase $A_{2}$ activity, if maintained in the neuritic outgrowth, could lead to a failure of 'synaptic pruning' and, potentially, predispose to the later onset of schizophrenia. There is also some evidence that antipsychotic medication reduces phospholipase $A_{2}$ activity to normal (Gattaz et al, 1987). These findings suggest a pathway open to diverse influences (genes, nutrition, toxins and experiences), which may account for disparate findings in schizophrenia and integrate competing causal models derived from pharmacology, epidemiology and clinical observation.

The fMRI and phospholipase $A_{2}$ data taken together encouraged enzyme geneticists to include regulators of lipid metabolism among candidate genes for schizophrenia. Hudson et al (1996) studied a poly-A repeat polymorphism associated with the gene for phospholipase $A_{2}$. They found that larger alleles were enriched in subjects with schizophrenia and suggested that these alleles may influence expression of the phospholipase $A_{2}$ family of genes, thus affecting phospholipase $\mathrm{A}_{2}$ activity. Although Price et al (1997) failed to replicate these findings, the regulation of phospholipase $A_{2}$ activity rather than the structure of the phospholipase $A_{2}$ gene family may prove to be of critical relevance.

\section{Neurotoxicity}

One of the more plausible of the many hypotheses of schizophrenia is that endogen- ous reactive oxygen species, released during normal respiration, damage nucleic acids and lipids, as has been shown in cancer (Duthie et al, 1996). This mechanism could reveal or amplify latent 'pre-schizophrenic' genetic abnormalities. Increased peroxidation could also account for the lowered PUFA concentrations seen in schizophrenia. McCreadie et al (1995) measured lipid peroxide and vitamin $\mathrm{E}$ in the blood of subjects with schizophrenia and controls. They found that subjects with schizophrenia have significantly higher lipid peroxide and lower vitamin E concentrations than controls matched for age, gender and smoking status. Patients who smoke show the effect more markedly. They could detect no effect of medication.

\section{LIPID METABOLISM AND DISEASE}

The importance of lipid metabolism in disease is beyond doubt. Cardiovascular mortality is reduced by lifestyle improvements such as cessation of smoking, increased exercise and better diet. The case remains to be established that lipids play an important role in the pathogenesis of psychiatric disorders. Important questions remain. The reliability and validity of many lipid measurements are not well proven; storage and handling effects are poorly studied. Diet is likely to be of major importance in determining lipid composition of cell membranes, possibly at two stages: around the time of measurement and during neurodevelopment. This would imply potential for gene-diet interaction in utero and during early childhood (McGill et al, 1996). There may be influences of psychological stressors on lipid measures, possibly mediated by hormonal mechanisms (especially adrenal and thyroid); endocrine function is known to be altered in acute psychosis (Whalley et al, 1989).

Medication and smoking effects are yet to be explored fully. Even so, the evidence of lipid dysfunction is compelling enough to postulate that PUFA and antioxidant supplements will help to relieve schizophrenia. Puri et al (1998) report a single case of schizophrenia responding to supplementation with eicosapentaenoic acid. There is evidence that better diet and cessation of smoking have a beneficial effect on lipid metabolism (Brown et al, 1991). Reduced dietary cholesterol consumption decreases the risk of heart disease. 
Enrichment with micronutrients such as eicosapentaenoic acid (Puri et al, 1998) may be helpful in schizophrenia, as Lorenzo oil can be in adrenoleucodystrophy (Moser, 1993). Improved maternal nutrition during pregnancy and breast-feeding may help to prevent neurodevelopmental abnormalities, much as folic acid supplementation in pregnancy reduces the incidence of spina bifida.

Molecular genetic techniques are likely to prove informative in the study of causes of schizophrenia. The evidence presented here supports the case for inclusion of genes concerned with the regulation of brain lipid metabolism in studies of families with schizophrenia in high density. Abnormalities in lipid metabolism may prove relevant to understanding neurodevelopmental abnormalities in schizophrenia and how obstetric complications and malnutrition increase its risk. Potentially, advances in lipid neurochemistry in schizophrenia will improve the choice of novel therapeutic strategies to ameliorate the public and personal burden of schizophrenia.

\section{REFERENCES}

Abdulla, Y. H. \& Hamadah, K. (1975) Effect of ADP on $P G E$, formation in blood platelets from patients with depression, mania and schizophrenia. British journal of Psychiotry. 127, 591-595.

Albers, M., Meurer, H., Marki, F., ef al (1993) Phospholipase A2 activity in serum of neuroleptic-naive psychiatric inpatients. Pharmocopsychiatry, 26.94-98.

Brown, A. J., Pang, E. \& Roberts, D. C. K. (1991) Persistent changes in the fatty acid composition of erythrocyte membranes after moderate intake of $n-3$ polyunsaturated fatty acids: study design implications. Amenican journal of Clinical Nutrition, 54, 668 673.

Carlson, S. E., Carver, J. D. \& House, S. G. (1986) High fat diets varying in ratios of polyunsaturated to saturated fatty acid and linolenic acid: a comparison of rat neural and red cell membrane phospholipids. journol of Nutrition, 116, 718-725

Crawford, M. A. (1993) The role of essential fatty acids in neural development: implications for perinatal nutrition. American journal of Clinical Nutrition, 57 (suppl.). 703-710.

Duthie, S. J., Ma, A., Ross, M. A., et al (1996) Antioxidant supplementation decreased oxidative DNA damage in human lymphocytes. Concer Research, 56. $1291-1295$.

NICHOLAS P.WALKER, MRCPsych, HELEN C. FOX, MA, LAWRENCE I. WHALIEY, FRCPsych, University of Aberdeen Medical School. Royal Cornhill Hospital, Aberdeen

Correspondence: Professor Lawrence J.Whalley, University of Aberdeen Medical School, Royal Cornhill Hospital. Aberdeen AB25 2ZH. e-mail: mental.health.CRC@abdn.ac.uk

(First received 30 March 1998, final revision 26 October 1998, accepted 2 November 1998)

Fischer, S., Kissling, W. \& Kuss, H. J. (1992) Schizophrenic patients treated with high dose phenothiazine or thioxanthene become deficient in polyunsaturated fatty acids in their thrombocytes. Biochemical Pharmocology, 44, 317-323.

Gattaz, W. F., Kollisch, M., Thuren, T., et al (1987) Increased plasma phospholipase-a2 activity in schizophrenic patients: reduction after neuroleptic therapy. Biological Psychiatry. 22, 421-426.

Glen, A. L., Glen, E. M., Horrobin, D. F., et al (1994)

A red cell membrane abnormality in a subgroup of schizophrenic patients: evidence for two diseases. Schizophrenio Research, 12, 53-61.

Hess, D. T., Patterson, S. L., Smith, D. S., et af (1993) Neuronal growth cone collapse and inhibition of protein fatty acylation by nitric oxide. Nature, 366. 562-565.

Horrobin, D. F. (1977) Schizophrenia as a prostaglandin deficiency disease. Loncet, i, 936-937.

_ , Manku, M. S., Hillman, H., et ol (1991) Fatty acid levels in the brains of schizophrenics and normal controls. Biological Psychiatry, 30. 795-805.

Hudson, C. J., Kennedy, J. L., Gotowiec, A., et of (1996) Genetic vanant near cytosolic phospholipase a2 associated with schizophrenia. Schizophrenio Research, 21. III-116.

Huttenlocher, P. (1979) Synaptic density in human frontal cor tex. developmental changes and effects of ageing, Brain Research, 163, 195-205.

Jones, P. \& Murray, R. M. (1991) The genetics of schizophrenia is the genetics of neurodevelopment. British journal of Psychiatry. 158, 615-623.

Lucas, A., Morley, R., Cole, T. J., et al (1992) Breast milk and subsequent intelligence quotient in children born preterm. Loncet, 339. 261-264.

Makrides, M., Neumann, M. A., Byand, R.W., et of (1994) Fatty acid composition of brain, retina and erythrocytes in breast- and formula-fed infants American journal of Clinical Nutriton. 60, 189-194.

McCreadle, R. G., MacDonald, E., Wiles, D., et at (1995) The Nithsdale schizophrenia surveys. XIV: Plasma lipid peroxide and serum vitamin $E$ levels in patients with and without tardive dyskinesia and in normal subjects. British journal of Psychiatry, 167 610-617

McGill, H. C., Mott, G. E., Lewis, D. S., et al (1996) Early determinants of adult regulation, effects of infant nutrition on adutt lipid and lipoprotein metabolism. Nutrition Revews, 54 (suppl.). $31-40$.
Mellor, J. E., Laugharne, J. D. E. \& Peet, M. (1996) Omega- 3 fatty acid supplementation in schizophrenic patients. Human Psychopharmocology. II. 39-46.

Moser, H.W. (1993) Lorenzo oil therapy for adrenoleukodystrophy: a prematurely amplified hope Annals of Neurology, 34, 121-122.

Negre-Aminou, P., Nemenoff, R. A., Wood, M. R., ef al (1996) Characterization of phospholipase A2 activity enriched in the nerve growth cone. Journal of Neurochemistry. 67, 2599-2608.

Peet, M., Laugharne, J. D., Horrobin, D. F., et al (1994) Arachidonic acid: a common link in the biology of schizophrenia? [Letter]. Archives of General Psychioury, 51. 665-666.

Pettegrew, J. W., Keshavan, M. S., Panchalingam, K. et al (1991) Alterations in brain high-energy phosphate and membrane phospholipid metabolism in firstepisode, drug-nave schizophrenics. A pilot study of the dorsal prefrontal cortex by in uno phosphorus 31 nuclear magnetic resonance spectroscopy. Archives of General Psychiotry, 48, 563-568.

Price, S., Fox, H., St Clair, D., ef ol (1997) Lack of association between schizophrenia and a polymorphism close to the cytosolic phospholipase A2 gene. Psychatic Genetics, 7. III-114.

Puri, B. K., Steiner, R. \& Richardson, A. J. (1998) Sustained remission of positive and negative symptoms of schizophrenia following treatment with eicosapentaenoic acid. Archives of General Psychiatry. 55. $188-189$.

Ross, B. M., Hudson, C., Erlich, J., et al (1997) Increased phospholipud breakdown in schizophrenia. evidence for the involvement of a calcium-independent phospholipase A2. Avchives of Generol Psychiotry, 54. $487-494$

Smalheiser, N. R., Dissanayake, S. Kapil, A. (1996) Rapid regulation of neurite outgrowth and retraction by phospholipase A2-derived arachidonic acid and its metabolites. Brain Research, 721. 39-48.

Stanley, J. A., Williamson, P. C., Drost, D. J., et al (1995) An in wo study of the prefrontal cortex of schizophrenic patients at different stages of illness va phosphorus magnetic resonance spectroscopy. Archives of General Psychiotry, 52. 399-406.

Whalley, L. J., Christie, J. E., Blackwood, D. H. R., et al (1989) Disturbed endocrine function in the psychoses, disordered horneostasis or disease process. Britush journal of Psychiatry. 155, 455-461. 